\title{
ERROR ESTIMATES FOR SCALAR CONSERVATION \\ LAWS BY A KINETIC APPROACH
}

\author{
A. OMRANE
}

Received 13 March 2006; Accepted 13 April 2006

We use the kinetic approach of Perthame and Tadmor (1991) to calculate the error estimates for general scalar conservation laws governing problems in gas dynamics or fluid mechanics in general. The Kružkov and Kuznetsov techniques are generalized to this method, and an error bound of order $\sqrt{\varepsilon}$ (where $\varepsilon$ is the mean free path) is obtained.

Copyright (c) 2006 Hindawi Publishing Corporation. All rights reserved.

\section{Introduction}

The kinetic approaches for scalar conservation laws have attracted attention for the last few years. Much work has been done in this field, however, important developments such as error estimates have not been totally investigated. The Perthame-Tadmor approach [16] is here used to derive the error bound for the following Cauchy problem of conservation laws:

$$
\begin{gathered}
\partial_{t} u+\sum_{i=1}^{N} \partial_{x_{i}}\left(A_{i}(u)\right)=0, \\
u(0, x)=u_{0}(x),
\end{gathered}
$$

where $(t, x) \in\left[0,+\infty\left[\times \mathbb{R}^{N}, u=u(t, x) \in \mathbb{R}\right.\right.$, and where the functions $A_{i}^{\prime}(\cdot)=a_{i}(\cdot)$ are locally Lipschitz in $\mathbb{R}$.

It is well known that there is a lack of regularity of the solutions for $t>0$, so that we generally look for weak solutions for (1.1)-(1.2). Moreover, there is a unique weak solution of physical interest, satisfying the additional Kružkov [10] entropy condition:

$$
\partial_{t}(|u-\ell|)+\sum_{i=1}^{N} \partial_{x_{i}}\left(\operatorname{sgn}(u-\ell)\left(A_{i}(u)-A_{i}(\ell)\right)\right) \leq 0 \quad \text { in } \mathscr{D}^{\prime}\left(\mathbb{R}^{N}\right),
$$

$\ell \in \mathbb{R}$ being any real scalar. For more details, see for example the classical book of Smoller [17] and the references therein. Now, the problem (1.1)-(1.3), which can be seen as 
a macroscopic interpretation of gas dynamics, is generally approached by the viscosity method or by methods of numerical purpose.

In this work, we are concerned with a simple microscopic model which is not only adapted to solve the problems of existence, uniqueness, and convergence for scalar conservation laws, but adapted to calculate the error of convergence as well.

Our method consists in using the Perthame-Tadmor model which is analogous to the models of the kinetic theory of gases. This is a BGK model derived from Bhatnagar et al. [1], and contains most of the basic properties of fluid dynamics, mainly conservation of mass, momentum, and energy (see the book by Bouchut et al. [2], and/or the book by Cercignani [6]). A function $f(t, x, v)$ where $v$ is "velocity" is considered. It represents the distribution of "molecules" located at $x$, having a speed $v$, at time $t$.

Let us recall that a discrete-time version of the Perthame-Tadmor approach [16] was previously and simultaneously proposed by Brenier [4, 5], by Giga and Miyakawa [9], and by Miyakawa [14]. In [16], the authors prove the convergence towards the unique solution of (1.1)-(1.3). We are concerned here with the exact error of convergence.

The paper is organized as follows. In Section 2, we introduce the method of Perthame and Tadmor, giving the main definitions and results. In Section 3, we develop an approximate entropy inequality with a precise "entropy" remainder. Then we give the error estimates for the corresponding conservation law based on the Kružkov-Kuznetsov techniques in Section 4. Section 5 concerns some parabolic case.

\section{Around the kinetic model}

In this section, we give the main definitions and results due to Perthame and Tadmor. In 1991, they proposed the following BGK (say pseudo-BGK) model:

$$
\begin{aligned}
\partial_{t} f_{\varepsilon}+\sum_{i=1}^{N} a_{i}(v) \partial_{x_{i}} f_{\varepsilon} & =\frac{1}{\varepsilon}\left[\chi_{\int_{v} f_{\varepsilon}(t, x, v) d v}-f_{\varepsilon}\right] \\
f_{\varepsilon}(0, x, v) & =f_{0}(x, v)
\end{aligned}
$$

as a kinetic interpretation of the conservation law (1.1)-(1.2) when $\varepsilon \rightarrow 0$ (see [16] and also Lions et al. [13]). The scalar function $f_{\varepsilon}=f_{\varepsilon}(t, x, v)$ is the kinetic solution, $v \in \mathbb{R}, \chi_{w}$ $(w \in \mathbb{R})$ is a signature function (the pseudo-Maxwellian), and $\varepsilon>0$ the mean free path in comparison with the Boltzmann theory. If such a solution $f_{\varepsilon}$ is defined, the approximate solution for (1.1) is simply the function

$$
u_{\varepsilon}(t, x)=\int_{v \in \mathbb{R}} f_{\varepsilon}(t, x, v) d v
$$

while the signature $\chi_{w}$ is given by

$$
\chi_{w}(v)=\left\{\begin{array}{ll}
\operatorname{sgn} w & \text { if }(w-v) v \geq 0, \\
0 & \text { otherwise }
\end{array}= \begin{cases}1 & \text { if } 0 \leq v \leq w \\
-1 & \text { if } w \leq v \leq 0 \\
0 & \text { else. }\end{cases}\right.
$$


Remark 2.1. We immediately deduce the following formulae:

$$
u_{\varepsilon}(t, x)=\int_{v \in \mathbb{R}} \chi_{u_{\varepsilon}(t, x)}(v) d v,
$$

and $|w|=\int_{v \in \mathbb{R}}\left|\chi_{w}(v)\right| d v$ for any $w \in \mathbb{R}$. Moreover,

$$
A_{i}(u)-A_{i}(w)=\int_{v} a_{i}(v)\left(\chi_{u}(v)-\chi_{w}(v)\right) d v, \quad 1 \leq i \leq N .
$$

2.1. The well-posedness of the kinetic problem. Consider the linear version of (2.1) (without the term $\chi_{u_{\varepsilon}}$ at the right-hand side). The classical method of characteristics involves solving the equations

$$
\begin{gathered}
\frac{d X}{d t}(t, v)=a(v), \\
X(0, v)=X_{0} .
\end{gathered}
$$

For $\phi(t)=f_{\varepsilon}(t, X(t), v)$, we have $d \phi / d t=(-t / \varepsilon) \phi$, that is, $\phi=C \cdot e^{-(1 / \varepsilon) t}$. With the varying constant method, the solution of (2.1) is of the following form:

$$
f_{\mathcal{\varepsilon}}(t, x, v)=e^{-t / \varepsilon} f_{\varepsilon}(0, x-\tau a(v), v)+\frac{1}{\varepsilon} \int_{\tau=0}^{t} e^{(\tau-t) / \varepsilon} \chi_{u_{\varepsilon}(\tau, x-(t-\tau) a(v))}(v) d \tau
$$

(see Omrane [15]). We also have the $L^{1}$ contraction

$$
\begin{aligned}
\| f_{\varepsilon}(t, x, v)-\left.g_{\varepsilon}(t, x, v)\right|_{L^{1}\left(\mathbb{R}_{x}^{N} \times \mathbb{R}_{v}\right)} \leq & e^{-t / \varepsilon}|| f_{\varepsilon}(0, x, v)-g_{\varepsilon}(0, x, v) \|_{L^{1}\left(\mathbb{R}_{x}^{N} \times \mathbb{R}_{v}\right)} \\
& +\frac{1}{\varepsilon} \int_{v} \int_{\tau=0}^{t} e^{(\tau-t) / \varepsilon}\left\|\chi_{u_{\varepsilon}(\tau, x)}(v)-\chi_{\omega_{\varepsilon}(\tau, x)}(v)\right\|_{L^{1}\left(\mathbb{R}_{x}^{N}\right)} d \tau d v,
\end{aligned}
$$

(here $w_{\varepsilon}=\int_{v} g_{\varepsilon}$ ) where $f_{\varepsilon}$ and $g_{\varepsilon}$ are two solutions of (2.1). This gives

$$
\begin{aligned}
\left\|f_{\varepsilon}(t, x, v)-g_{\varepsilon}(t, x, v)\right\|_{L^{1}\left(\mathbb{R}^{N} \times \mathbb{R}_{v}\right)} \leq & e^{-t / \varepsilon}\left\|f_{\varepsilon}(0, x, v)-g_{\varepsilon}(0, x, v)\right\|_{L^{1}\left(\mathbb{R}^{N} \times \mathbb{R}_{v}\right)} \\
& +\left(1-e^{-t / \varepsilon}\right) \sup _{0 \leq \tau \leq t}\left\|f_{\varepsilon}(\tau, x, v)-g_{\varepsilon}(\tau, x, v)\right\|_{L^{1}\left(\mathbb{R}^{N} \times \mathbb{R}_{v}\right)} .
\end{aligned}
$$

Since $0<\left(1-e^{-t / \varepsilon}\right)<1$ if $f_{\varepsilon}(0, x, v)=g_{\varepsilon}(0, x, v)$, then there is a unique solution to (2.1). Moreover, by (2.10), the kinetic solution is continuously dependent on the initial data. We also have the contraction inequality

$$
\left\|f_{\varepsilon}(t, x, v)-g_{\mathcal{\varepsilon}}(t, x, v)\right\|_{L^{1}\left(\mathbb{R}^{N} \times \mathbb{R}_{v}\right)} \leq\left\|f_{\mathcal{\varepsilon}}(0, x, v)-g_{\mathcal{E}}(0, x, v)\right\|_{L^{1}\left(\mathbb{R}^{N} \times \mathbb{R}_{v}\right)} .
$$

Theorem 2.2. The model (2.1) is well-posed in $L^{\infty}\left([0, T] ; L^{1}\left(\mathbb{R}^{N} \times \mathbb{R}_{v}\right)\right)$, and the inequality of contraction (2.11) holds true.

Proof. See the article of Perthame and Tadmor [16] for the proof. 
4 Error bounds to conservation laws by kinetic approaches

2.2. Finite speed of propagation. In [16], we find the following. We first notice that for any $t \in[0, T]$,

$$
\left\|u_{\varepsilon}(t, \cdot)\right\|_{L^{\infty}\left(\mathbb{R}^{N}\right)} \leq \int_{v}\left\|f_{\varepsilon}(t, \cdot, v)\right\|_{L^{\infty}\left(\mathbb{R}^{N}\right)} d v \leq \int_{v}\left\|f_{\varepsilon}(0, \cdot, v)\right\|_{L^{\infty}\left(\mathbb{R}^{N}\right)} d v
$$

with the help of Jensen's inequality. Denoting $u_{\infty}=\left\|u_{\varepsilon}(t, x)\right\|_{L^{\infty}\left(\mathbb{R}_{x}^{N}\right)}$, the support of $f_{\varepsilon}(t$, $x, \cdot)$ is showed to be contained in $K_{v}$ :

$$
K_{v}=\left(\operatorname{supp}\left(f_{\varepsilon}(0, x, \cdot)\right)\right) \cup\left[-u_{\infty},+u_{\infty}\right]
$$

Consequently, the term $a(v) \cdot \nabla_{x} f_{\varepsilon}$ is also supported in $K_{v}$, and the elements $\left|a_{i}(v)\right|$ are bounded by

$$
a_{\infty}=\max \left\{\left|a_{i}(v)\right|, v \in K_{v}, i=1, \ldots, N\right\} .
$$

Remark 2.3. In fact, the support of $f_{\varepsilon}(t, x, \cdot)$ is mainly contained in $\left[-u_{\infty},+u_{\infty}\right]$, because of the decay of the initial data in (2.8).

2.3. The kinetic entropies $H\left(f_{\varepsilon}\right)$. The notion of "kinetic" entropy has the same sense as the classical entropy for conservation laws. It is just a microscopic version of it, and the name is due to the presence of the speed vector $a(v)$.

Definition 2.4. The kinetic entropies are the functions $H\left(f_{\varepsilon}\right)$ such that for any positive test function $\varphi \in \mathscr{D}\left(\mathbb{R}_{+}^{t} \times \mathbb{R}_{x}^{N}\right)$,

$$
-\int_{0}^{+\infty} \int_{\mathbb{R}^{N}} \int_{\mathbb{R}_{v}}\left(\left[\partial_{t}+a(v) \nabla_{x}\right] \varphi\right) H\left(f_{\varepsilon}\right) d v d x d t \leq 0
$$

(according to the $H$-theorem in Boltzmann theory).

Multiplying (2.1) by $\operatorname{sgn}\left(f_{\varepsilon}-\chi_{\ell}\right), \ell \in \mathbb{R}$, and integrating by parts, we have

$$
\begin{aligned}
& -\int_{0}^{+\infty} \int_{\mathbb{R}^{N}} \int_{\mathbb{R}_{v}}\left(\left[\partial_{t}+a(v) \nabla_{x}\right] \varphi\right)\left|f_{\varepsilon}-\chi_{\ell}\right| d v d x d t \\
& \quad=-\frac{1}{\varepsilon} \int_{0}^{+\infty} \int_{\mathbb{R}^{N}} \int_{\mathbb{R}_{v}}\left(f_{\varepsilon}-\chi_{\ell}+\chi_{\ell}-\chi_{u_{\varepsilon}}\right) \varphi \operatorname{sgn}\left(f_{\varepsilon}-\chi_{\ell}\right) d v d x d t \\
& \quad \leq-\frac{1}{\varepsilon} \int_{0}^{+\infty} \int_{\mathbb{R}^{N}} \int_{\mathbb{R}_{v}}\left|f_{\varepsilon}-\chi_{\ell}\right| \varphi d v d x d t+\int_{0}^{+\infty} \int_{\mathbb{R}^{N}} \frac{1}{\varepsilon}\left|u_{\varepsilon}-\ell\right| \varphi d x d t \leq 0,
\end{aligned}
$$

since $\left|u_{\varepsilon}-\ell\right|=\left|\int_{v} f_{\varepsilon}-\chi_{\ell} d v\right|$.

The functions $H\left(f_{\varepsilon}\right)=\left|f_{\varepsilon}-\chi_{\ell}\right|$ are the kinetic entropies. Notice that $\ell$ is any given scalar (it may also be any function uniformly bounded in $t$ and $x$ ). 


\section{The approximate entropy solutions}

In this section, we show that the Kružkov method can be applied. Let $(s, y) \in(0, T) \times \mathbb{R}^{N}$ be the new variables, we then define $Q=(0, T)^{2} \times \mathbb{R}_{x}^{N} \times \mathbb{R}_{y}^{N}$ and $\varphi=\varphi(t, s, x, y)$.

Proposition 3.1. Let $u=u(s, y)$ be the unique entropy solution of (1.1)-(1.3), and $f_{\varepsilon} \in$ $L^{\infty}\left([0, T] ; L^{1}\left(\mathbb{R}^{N} \times \mathbb{R}_{v}\right)\right)$ the approximate kinetic solution given by (2.1). Then

$$
\begin{aligned}
& -\int_{Q}\left|u_{\varepsilon}(t, x)-u(s, y)\right|\left(\left(\partial_{t}+\partial_{s}\right) \varphi\right) d Q \\
& -\int_{Q} \operatorname{sgn}\left(u_{\varepsilon}-u\right)\left(A\left(u_{\varepsilon}(t, x)\right)-A(u(s, y))\right)\left(\left(\nabla_{x}+\nabla_{y}\right) \varphi\right) d Q \\
& \leq \int_{Q} \int_{v}\left|\partial_{t} \varphi\right|\left|\chi_{u_{\varepsilon}}-f_{\varepsilon}\right| d v d Q+a_{\infty} \int_{Q} \int_{v}\left|\nabla_{x} \varphi\right|\left|\chi_{u_{\varepsilon}}-f_{\varepsilon}\right| d v d Q
\end{aligned}
$$

for any test function $\varphi \in \mathscr{D}(Q)$.

Proof. Recall that $\ell \in \mathbb{R}$ can be chosen as any $\ell=\ell(s, y)$ for example, uniformly bounded with respect to $s$ and $y$. We write

$$
\begin{aligned}
-\int_{Q} \int_{v} & \left(\left[\partial_{t}+a(v) \nabla_{x}\right] \varphi\right)\left|\chi_{u_{\varepsilon}}-\chi_{\ell}\right| d v d Q \\
= & -\int_{Q} \int_{v}\left(\left[\partial_{t}+a(v) \nabla_{x}\right] \varphi\right)\left(\left|\chi_{u_{\varepsilon}}-\chi_{\ell}\right|-\left|f_{\varepsilon}-\chi_{\ell}\right|\right) d v d Q \\
& -\int_{Q} \int_{v}\left(\left[\partial_{t}+a(v) \nabla_{x}\right] \varphi\right)\left|f_{\varepsilon}-\chi_{\ell}\right| d v d Q .
\end{aligned}
$$

With the definition on the kinetic entropies and the following triangle inequality:

$$
|| \chi_{u_{\varepsilon}}-\chi_{\ell}|-| f_{\varepsilon}-\chi_{\ell}|| \leq\left|\chi_{u_{\varepsilon}}-f_{\varepsilon}\right|
$$

we obtain

$$
-\int_{Q} \int_{v}\left(\left[\partial_{t}+a(v) \nabla_{x}\right] \varphi\right)\left|\chi_{u_{\varepsilon}}-\chi_{\ell}\right| d v d Q \leq \int_{Q} \int_{v}\left|\left[\partial_{t}+a(v) \nabla_{x}\right] \varphi\right|\left|\chi_{u_{\varepsilon}}-f_{\varepsilon}\right| d v d Q .
$$

Using Remark 2.1, and the finite speed of propagation property (2.14), we deduce

$$
\begin{aligned}
& -\int_{Q}\left|u_{\varepsilon}(t, x)-\ell(s, y)\right| \partial_{t} \varphi d Q \\
& \quad-\int_{Q} \operatorname{sgn}\left(u_{\varepsilon}-\ell\right)\left(A\left(u_{\varepsilon}(t, x)\right)-A(\ell(s, y))\right) \nabla_{x} \varphi d Q \\
& \leq \int_{Q} \int_{v}\left|\partial_{t} \varphi\right|\left|\chi_{u_{\varepsilon}}-f_{\varepsilon}\right| d v d Q+a_{\infty} \int_{Q} \int_{v}\left|\nabla_{x} \varphi\right|\left|\chi_{u_{\varepsilon}}-f_{\varepsilon}\right| d v d Q .
\end{aligned}
$$


6 Error bounds to conservation laws by kinetic approaches

From another side, the exact entropy solution $u(s, y)$ of $(1.1)-(1.3)$ verifies for all $\ell=$ $\ell(t, x) \in L^{\infty}\left(\mathbb{R}_{+} \times \mathbb{R}_{x}^{N}\right)$ that

$$
\begin{aligned}
& -\int_{Q}|u(s, y)-\ell(t, x)| \partial_{s} \varphi d Q \\
& \quad-\int_{Q} \operatorname{sgn}(u(s, y)-\ell(t, x))(A(u(s, y))-A(\ell(t, x))) \nabla_{y} \varphi d Q \leq 0 .
\end{aligned}
$$

If we replace $\ell(s, y)$ by $u(s, y)$ (the exact solution) in (3.5), and $\ell(t, x)$ by $u_{\varepsilon}(t, x)$ in (3.6), then we sum up to obtain (3.1).

3.1. The test functions. We define $\varphi$ as follows:

$$
\varphi(t, s, x, y)=\psi_{\eta}\left(\frac{t+s}{2}\right) \theta_{h}(t-s) \prod_{i=1}^{N} \theta_{h}\left(x_{i}-y_{i}\right),
$$

where $\psi_{\eta}$ is the characteristic function defined by

$$
\psi_{\eta}(t)=\chi^{\eta}\left(\frac{t-t_{0}}{\Delta t}\right), \quad \chi^{\eta}(\tau)=\int_{0}^{\tau} \theta_{\eta}(s) d s+\int_{\tau}^{1} \theta_{\eta}(s-1) d s,
$$

and where the function $\theta_{h}$ is the classical mollifier. For $h>0$, we also have $-h \leq \theta_{h}(\cdot) \leq h$, $\int_{-h}^{h} \theta_{h}(s) d s=1, \theta_{h}(s)=(1 / h) \theta(s / h)$, where $\theta$ is a regularized Dirac function of support $[-1,1]: \int_{-1}^{1} \theta(s) d s=1$. We have the following corollary.

Corollary 3.2. Under the hypothesis of Proposition 3.1, the following entropy inequality holds:

$$
\begin{aligned}
& -\int_{Q}\left|u_{\varepsilon}(t, x)-u(s, y)\right|\left[\psi_{\eta}\right]^{\prime}\left(\frac{t+s}{2}\right) \theta_{h}(t-s) \prod_{i=1}^{N} \theta_{h}\left(x_{i}-y_{i}\right) d Q \\
& \quad \leq \int_{Q} \int_{v}\left|\partial_{t} \varphi\right|\left|\chi_{u_{\varepsilon}}-f_{\varepsilon}\right| d v d Q+a_{\infty} \int_{Q} \int_{v}\left|\nabla_{x} \varphi\right|\left|\chi_{u_{\varepsilon}}-f_{\varepsilon}\right| d v d Q .
\end{aligned}
$$

Proof. To get (3.9), it is convenient to choose $\varphi$ as in $(3.7)$ (see $[15,18]$ ). With this, we obtain

$$
\begin{gathered}
\partial_{x_{i}} \varphi+\partial_{y_{i}} \varphi=0, \quad \text { for } i=1, \ldots, N, \\
\partial_{t} \varphi+\partial_{s} \varphi=\left[\psi_{\eta}\right]^{\prime}\left(\frac{t+s}{2}\right) \theta_{h}(t-s) \prod_{i=1}^{N} \theta_{h}\left(x_{i}-y_{i}\right),
\end{gathered}
$$

hence we have the left-hand side of (3.9).

3.2. Bounds. Let us begin with some properties of the test function. For $t_{0}$ and $t_{1}$ in $[0, T]$, we have

$$
\iint_{t_{0}}^{t_{1}}\left|\psi_{\eta}^{\prime}\left(\frac{t+s}{2}\right)\right| \theta_{h}(t-s) d t d s \leq 1
$$


Indeed, note that

$$
\psi_{\eta}^{\prime}\left(\frac{t+s}{2}\right)=\frac{1}{\Delta t}\left[\theta_{\eta}\left(\frac{(t+s) / 2-t_{0}}{\Delta t}\right)-\theta_{\eta}\left(\frac{(t+s) / 2-t_{1}}{\Delta t}\right)\right]
$$

Moreover, we easily see that

$$
\begin{aligned}
& \iint_{t_{0}}^{t_{1}} \theta_{\eta}\left(\frac{(t+s) / 2-t_{i}}{\Delta t}\right) \theta_{h}(t-s) d t d s \\
& \quad=2 \Delta t \int_{t_{0}}^{t_{1}} \int_{t_{0}-s}^{t_{1}-s} \theta_{2 \Delta t \eta}\left(\tau+2 s-2 t_{i}\right) \theta_{h}(\tau) d \tau d s=\frac{1}{2}, \quad \text { for } i=0,1 .
\end{aligned}
$$

In particular (3.11) holds, and we have the lemma.

Lemma 3.3. Suppose that $f_{\varepsilon}(0, x, v) \in L^{1}\left(\mathbb{R} ; B V\left(\mathbb{R}^{N}\right)\right)$, which is compactly supported in $\mathbb{R}_{v}$. Let $u(s, y)$ be the entropy solution of $(1.1)-(1.3)$, and $u_{\varepsilon}(t, x)$ the approximate solution given by (2.3), then

$$
\begin{aligned}
& -\int_{Q}\left|u(s, y)-u_{\varepsilon}(t, x)\right|\left[\psi_{\eta}\right]^{\prime}\left(\frac{t+s}{2}\right) \theta_{h}(t-s) d Q \\
& \quad \leq\left(\frac{1}{2}+\frac{N a_{\infty}}{h}+\frac{\left(1+a_{\infty}\right)}{h}\left|\theta^{\prime}\right|_{L^{1}(\mathbb{R})}\left|\psi_{\eta}\right|_{L^{1}(\mathbb{R})}\right) \sup _{0 \leq t \leq T} \| f_{\varepsilon}-\left.\chi_{u_{\varepsilon}}\right|_{L^{1}\left(\mathbb{R}_{x}^{N} \times \mathbb{R}_{v}\right)} .
\end{aligned}
$$

Proof. Using (3.11), we write

$$
\begin{aligned}
& \int_{Q} \int_{v}\left|\partial_{t} \varphi\right|\left|f_{\varepsilon}-\chi_{u_{\varepsilon}}\right| d v d Q \\
& \leq \int_{x}\left(\int_{y} \prod_{i=1}^{N} \theta_{h}\left(x_{i}-y_{i}\right) d y\right) \int_{t} \int_{s}\left\{\frac{1}{2}\left|\psi_{\eta}^{\prime}\left(\frac{t+s}{2}\right)\right| \theta_{h}(t-s)\right. \\
& \left.+\frac{1}{h^{2}} \psi_{\eta}\left(\frac{t+s}{2}\right) \theta^{\prime}\left(\frac{t-s}{h}\right)\right\} d s d t \int_{v}\left|f_{\varepsilon}-\chi_{u_{\varepsilon}}\right| d v d x \\
& \leq \sup _{0 \leq t \leq T} \int_{x} \int_{v}\left|f_{\varepsilon}-\chi_{u_{\varepsilon}}\right| d v d x\left\{\frac{1}{2} \int_{t} \int_{s}\left|\psi_{\eta}^{\prime}\left(\frac{t+s}{2}\right)\right| \theta_{h}(t-s) d s d t\right. \\
& \left.+\frac{1}{h^{2}} \int_{t} \int_{s} \psi_{\eta}\left(\frac{t+s}{2}\right) \theta^{\prime}\left(\frac{t-s}{h}\right) d s d t\right\} \\
& \leq \sup _{0 \leq t \leq T}|| f_{\varepsilon}-\left.\chi_{u_{\varepsilon}}\right|_{L^{1}\left(\mathbb{R}_{x}^{N} \times \mathbb{R}_{v}\right)}\left[\frac{1}{2} \times 1+\frac{1}{h^{2}} \times h\left|\theta^{\prime}\right|_{L^{1}(\mathbb{R})}\left|\psi_{\eta}\right|_{L^{1}(\mathbb{R})}\right],
\end{aligned}
$$


8 Error bounds to conservation laws by kinetic approaches

and

$$
\begin{aligned}
& \int_{Q} \int_{v}\left|\nabla_{x} \varphi\right|\left|f_{\varepsilon}-\chi_{u_{\varepsilon}}\right| d v d Q \\
& \leq\left[\frac{N}{h}+\frac{1}{h}\left(\left|\psi_{\eta}\right|_{L^{1}(\mathbb{R})}\left|\theta^{\prime}\right|_{L^{1}(\mathbb{R})}\right)\right] \sup _{0 \leq t \leq T}|| f_{\varepsilon}-\left.\chi_{u_{\varepsilon}}\right|_{L^{1}\left(\mathbb{R}_{x}^{N} \times \mathbb{R}_{v}\right)},
\end{aligned}
$$

with the same arguments.

We now control the term $\sup _{0 \leq t \leq T}\left\|f_{\varepsilon}-\chi_{u_{\varepsilon}}\right\|_{L^{1}\left(\mathbb{R}_{x}^{N} \times \mathbb{R}_{v}\right)}$ at the right-hand side of (3.14). Proposition 3.4. Consider the kinetic initial data $f_{\varepsilon}(0, x, v) \in L^{1}\left(\mathbb{R}_{v} ; \mathrm{BV}\left(\mathbb{R}^{N}\right)\right)$ which is compactly supported in $\mathbb{R}_{v}$. Then

$$
\begin{aligned}
& \left\|f_{\varepsilon}(t, x, v)-\chi_{u_{\varepsilon}(t, x)}(v)\right\|_{L^{1}\left(\mathbb{R}^{N} \times \mathbb{R}_{v}\right)} \\
& \leq 2 \varepsilon a_{\infty}\left\|f_{\varepsilon}(0, x, v)\right\|_{\mathrm{BV}\left(\mathbb{R}^{N} ; L^{1}\left(\mathbb{R}_{v}\right)\right)}+2\left\|f_{\varepsilon}(0, x, v)-\chi_{u_{\varepsilon}(0, x)}(v)\right\|_{L^{1}\left(\mathbb{R}^{N} \times \mathbb{R}_{v}\right)},
\end{aligned}
$$

for every $t>0$, where

$$
\left\|f_{\varepsilon}(0, x, v)\right\|_{\mathrm{BV}\left(\mathbb{R}^{N} ; L^{1}\left(\mathbb{R}_{v}\right)\right)}=\sup _{|\Delta x| \neq 0} \int_{x} \int_{v} \frac{\left|f_{\varepsilon}(0, x+\Delta x, v)-f_{\varepsilon}(0, x, v)\right|}{|\Delta x|} d x d v .
$$

Proof. Suppose that $\left\|f_{\varepsilon}(0, x, v)\right\|_{\mathrm{BV}\left(\mathbb{R}^{N} ; L^{1}\left(\mathbb{R}_{v}\right)\right)}$ is uniformly bounded in $\varepsilon$, then using the contraction inequality $(2.11)$, the corresponding solution $f_{\varepsilon}(t, x, v)$ satisfies that for every $0 \leq t \leq T$,

$$
\left\|f_{\varepsilon}(t, x, v)\right\|_{\mathrm{BV}\left(\mathbb{R}^{N} ; L^{1}\left(\mathbb{R}_{v}\right)\right)} \leq\left\|f_{\varepsilon}(0, x, v)\right\|_{\mathrm{BV}\left(\mathbb{R}^{N} ; L^{1}\left(\mathbb{R}_{v}\right)\right)} .
$$

Moreover,

$$
\chi_{u_{\varepsilon}(t, x)(v)}-f_{\varepsilon}(t, x, v)=\varepsilon\left\{\frac{\partial}{\partial t} f_{\varepsilon}(t, x, v)+a(v) \cdot \nabla_{x} f_{\varepsilon}(t, x, v)\right\} \in L^{1}\left(\mathbb{R}_{x}^{N} \times \mathbb{R}_{v}\right),
$$

that is for every $t \geq 0$ and every $\varepsilon>0$,

$$
\left\|\chi_{u_{\varepsilon}(t, x)}(v)-f_{\mathcal{\varepsilon}}(t, x, v)\right\|_{L^{1}\left(\mathbb{R}^{N} \times \mathbb{R}_{v}\right)} \leq \varepsilon \mid\left\|\frac{\partial f_{\varepsilon}}{\partial t}(t, x, v)+a(v) \nabla_{x} f_{\mathcal{\varepsilon}}(t, x, v)\right\|_{L^{1}\left(\mathbb{R}^{N} \times \mathbb{R}_{v}\right)} .
$$

It is important to notice that we do not necessarily recover the $L^{1}$ regularity of the term $\partial f_{\varepsilon} / \partial t$ since

$$
\frac{\partial f_{\varepsilon}}{\partial t}=\frac{1}{\varepsilon}\left[\chi_{u_{\varepsilon}}-f_{\varepsilon}\right]-a(v) \cdot \nabla_{x} f_{\varepsilon},
$$

and not for $a(v) \cdot \nabla_{x} f_{\varepsilon}$ either. The quantities $\left|\partial f_{\varepsilon} / \partial t\right|$ and $\left|\partial f_{\varepsilon} / \partial x_{i}\right|$ for $1 \leq i \leq N$ are only measures that we can bound by the BV norm. Indeed, for $\varphi \in \mathscr{D}$, denote by $\|\cdot\|_{\mu}$ the norm

$$
\left\|f_{\varepsilon}\right\|_{\mathcal{M}}:=\sup _{\|\varphi\| \equiv 1}\left|\int_{v} \int_{z} \frac{\partial f_{\varepsilon}}{\partial z} \varphi d v d z\right|
$$


where $z=t, x_{i}$, for $i=1, \ldots, N$. Then we can write

$$
\left\|f_{\varepsilon}\right\|_{\mathcal{M}}=\sup _{\|\varphi\| \equiv 1}\left|\int_{v}\left(\int_{z} f_{\varepsilon} \varphi^{\prime}(z) d z\right) d v\right| \leq\left\|f_{\varepsilon}\right\|_{L^{1}\left(\mathbb{R}_{v} ; \mathrm{BV}(z)\right)} .
$$

Using this definition together with the finite speed of propagation property (2.14), we obtain

$$
\int_{x} \int_{v}\left|a(v) \nabla_{x} f_{\varepsilon}(t, x, v)\right| \leq a_{\infty}\left\|f_{\varepsilon}\right\|_{\mathcal{M}} \leq a_{\infty}\left\|f_{\varepsilon}(t, x, v)\right\|_{L^{1}\left(\mathbb{R}_{v} ; \mathrm{BV}\left(\mathbb{R}^{N}\right)\right)} .
$$

By (3.22) and (3.24), we also deduce

$$
\begin{aligned}
\int_{\Omega} \int_{v}\left|\frac{\partial f_{\varepsilon}}{\partial t}(t)\right| & \leq\left\|f_{\mathcal{\varepsilon}}(t)\right\|_{\mathrm{BV}(t)} \leq\left\|f_{\mathcal{\varepsilon}}(0, x, v)\right\|_{L^{1}\left(\mathbb{R}_{x}^{N} \times \mathbb{R}_{v} ; \mathrm{BV}(0, T)\right)} \\
& \leq a_{\infty}\left\|f_{\mathcal{\varepsilon}}(0, x, v)\right\|_{L^{1}\left(\mathbb{R}_{v} ; \mathrm{BV}\left(\mathbb{R}^{N}\right)\right)}+\left\|\chi_{u_{\varepsilon}(0, x)}(v)-f_{\mathcal{\varepsilon}}(0, x, v)\right\|_{L^{1}} .
\end{aligned}
$$

That is for all $T>0$ and every $0 \leq t \leq T$, we have

$$
\begin{aligned}
\left\|f_{\varepsilon}(t, x, v)-\chi_{u_{\varepsilon}}(v)\right\|_{L^{1}\left(\mathbb{R}_{x}^{N} \times \mathbb{R}_{v}\right)} \leq & 2 \varepsilon a_{\infty}\left\|f_{\varepsilon}(0, x, v)\right\|_{L^{1}\left(\mathbb{R}_{v} ; \operatorname{BV}\left(\mathbb{R}^{N}\right)\right)} \\
& +\left\|f_{\mathcal{\varepsilon}}(0, x, v)-\chi_{u_{\varepsilon}(0, x)}(v)\right\|_{L^{1}\left(\mathbb{R}_{x}^{N} \times \mathbb{R}_{v}\right)} .
\end{aligned}
$$

Remark 3.5. Denote $u_{\varepsilon}(0, x) \equiv u_{\varepsilon 0}(x):=u_{\varepsilon 0}$. Then, it is not restrictive to replace $\lim _{\varepsilon \rightarrow 0}$ $\left\|f_{\varepsilon}(0, x, v)-\chi_{u_{\varepsilon} 0}(v)\right\|_{L^{1}\left(\mathbb{R}_{x}^{N} \times \mathbb{R}_{v}\right)}=0$ by $f_{\varepsilon}(0, x, v)=\chi_{u_{\varepsilon} 0}(v)$ in the above proposition. This is useful for the applications.

We then have the proposition on the error bound.

Proposition 3.6. Suppose that $f_{\varepsilon}(0, x, v) \in L^{1}\left(\mathbb{R} ; B V\left(\mathbb{R}^{N}\right)\right)$, which is compactly supported in $\mathbb{R}_{v}$, and that initially $f_{\varepsilon}(0, x, v)=\chi_{u_{\varepsilon 0}}(v)$. Let $u(s, y)$ be the entropy solution of $(1.1)-(1.3)$, and $u_{\varepsilon}(t, x)$ the approximate solution given by (2.3). Then

$$
-\int_{Q}\left|u(s, y)-u_{\varepsilon}(t, x)\right|\left[\psi_{\eta}\right]^{\prime}\left(\frac{t+s}{2}\right) \theta_{h}(t-s) d Q \leq M \frac{\varepsilon}{h}\left\|u_{\varepsilon 0}\right\|_{\mathrm{BV}\left(\mathbb{R}^{N}\right)},
$$

where

$$
M=a_{\infty}\left(h+2 N a_{\infty}+2\left(1+a_{\infty}\right)\left|\theta^{\prime}\right|_{L^{1}(\mathbb{R})}\left|\psi_{\eta}\right|_{L^{1}(\mathbb{R})}\right) .
$$

Proof. If we take the simplified initial data form (Maxwellian data) of Remark 3.5, then Proposition 3.4 gives

$$
\left\|f_{\varepsilon}(t, x, v)-\chi_{u_{\varepsilon}(t, x)}(v)\right\|_{L^{1}\left(\mathbb{R}^{N} \times \mathbb{R}_{v}\right)} \leq 2 \varepsilon a_{\infty}\left\|f_{\varepsilon}(0, x, v)\right\|_{L^{1}\left(\mathbb{R}_{v} ; \mathrm{BV}\left(\mathbb{R}^{N}\right)\right)} .
$$

Moreover, noticing that

$$
\left\|\left.f_{\varepsilon}(0, x, v)\right|_{L^{1}\left(\mathbb{R}_{v} ; \mathrm{BV}\left(\mathbb{R}^{N}\right)\right)}=\right\| u_{\varepsilon}(0, x)\left\|_{\mathrm{BV}\left(\mathbb{R}^{N}\right)}=\right\| u_{\varepsilon 0}(x) \|_{\mathrm{BV}\left(\mathbb{R}^{N}\right)},
$$

we immediately deduce the result from Lemma 3.3. 
10 Error bounds to conservation laws by kinetic approaches

\section{Generalization to the Kuznetsov technique}

In this section, we will estimate the difference

$$
\left\|u\left(t_{1}, \cdot\right)-u_{\varepsilon}\left(t_{1}, \cdot\right)\right\|_{L^{1}\left(\mathbb{R}^{N}\right)}-\left\|u\left(t_{0}, \cdot\right)-u_{\varepsilon}\left(t_{0}, \cdot\right)\right\|_{L^{1}\left(\mathbb{R}^{N}\right)},
$$

where $t_{0}$ and $t_{1}$ are in $[0, T], T>0$. The techniques we will use are not new (see, e.g., Bouchut and Perthame [3]), and are known as the Kuznetsov techniques (see Kuznetsov [11], and Kuznetsov and Volshin [12]). The new thing here is the generalization of these techniques to the kinetic approach of Perthame and Tadmor, as the approximate solution is obtained from the kinetic model. We prepare by the lemma.

Lemma 4.1. Suppose that $f_{\varepsilon}(0, x, v) \in L^{1}\left(\mathbb{R} ; B V\left(\mathbb{R}^{N}\right)\right)$ is compactly supported in $v$, and that $u_{\varepsilon 0}(x)=u_{0}(x)$. Then

$$
\|u(t, \cdot)\|_{\mathrm{BV}\left(\mathbb{R}^{N}\right)} \leq\left\|u_{0}\right\|_{\mathrm{BV}\left(\mathbb{R}^{N}\right)}, \quad\left\|u_{\varepsilon}(t, \cdot)\right\|_{\mathrm{BV}\left(\mathbb{R}^{N}\right)} \leq\left\|u_{0}\right\|_{\mathrm{BV}\left(\mathbb{R}^{N}\right)}
$$

for every $t \in[0, T]$. Moreover, $u$ and $u_{\varepsilon}$ belong to the $\mathrm{BV}\left([0, T] ; L^{1}\left(\mathbb{R}^{N}\right)\right)$ for every $T>0$.

Proof. The proof is classical and relies on the translation property in $x$ and the contraction (2.11).

Proposition 4.2. Suppose that the hypothesis of Proposition 3.6 is satisfied. Then

$$
\begin{aligned}
I= & -\int_{Q}\left|u(s, y)-u_{\varepsilon}(t, x)\right|\left[\psi_{\eta}\right]^{\prime}\left(\frac{t+s}{2}\right) \theta_{h}(t-s) d Q \\
\geq & \left\|u\left(t_{1}, x\right)-u_{\varepsilon}\left(t_{1}, x\right)\right\|_{L^{1}\left(\mathbb{R}^{N}\right)}-\left\|u\left(t_{0}, x\right)-u_{\varepsilon}\left(t_{0}, x\right)\right\|_{L^{1}\left(\mathbb{R}^{N}\right)} \\
& -\left(4\left\|u_{0}\right\|_{\mathrm{BV}\left(\mathbb{R} ; L^{1}\left(\mathbb{R}^{N}\right)\right)}+2\left\|u_{0}\right\|_{\mathrm{BV}\left(\mathbb{R}^{N}\right)}\right) h .
\end{aligned}
$$

Proof. We split the integral $I$ using (3.12) into $I=I_{0}+I_{1}$ relatively to $t_{0}$ and $t_{1}$, where

$$
\begin{aligned}
& I_{0}=-\int_{Q}\left|u(s, y)-u_{\varepsilon}(t, x)\right| \frac{1}{\Delta t} \theta_{\eta}\left(\frac{(t+s) / 2-t_{0}}{\Delta t}\right) \theta_{h}(t-s) \prod_{i=1}^{N} \theta_{h}\left(x_{i}-y_{i}\right) d Q, \\
& I_{1}=+\int_{Q}\left|u(s, y)-u_{\varepsilon}(t, x)\right| \frac{1}{\Delta t} \theta_{\eta}\left(\frac{(t+s) / 2-t_{1}}{\Delta t}\right) \theta_{h}(t-s) \prod_{i=1}^{N} \theta_{h}\left(x_{i}-y_{i}\right) d Q .
\end{aligned}
$$

For $t=t_{0}$, we use the triangle inequality

$$
\begin{aligned}
\left|u(s, y)-u_{\varepsilon}(t, x)\right| \leq & \left|u(s, y)-u\left(t_{0}, y\right)\right|+\left|u\left(t_{0}, y\right)-u\left(t_{0}, x\right)\right| \\
& +\left|u\left(t_{0}, x\right)-u_{\varepsilon}\left(t_{0}, x\right)\right|+\left|u_{\varepsilon}\left(t_{0}, x\right)-u_{\varepsilon}(t, x)\right|,
\end{aligned}
$$

which also may be written as

$$
\begin{aligned}
-\left|u\left(t_{0}, x\right)-u_{\varepsilon}\left(t_{0}, x\right)\right| \leq & \left|u(s, y)-u\left(t_{0}, y\right)\right|+\left|u\left(t_{0}, y\right)-u\left(t_{0}, x\right)\right| \\
& +\left|u_{\varepsilon}\left(t_{0}, x\right)-u_{\varepsilon}(t, x)\right|-\left|u(s, y)-u_{\varepsilon}(t, x)\right| .
\end{aligned}
$$


We then integrate as follows:

$$
\begin{aligned}
-\int_{Q} \mid & u\left(t_{0}, x\right)-u_{\varepsilon}\left(t_{0}, x\right) \mid \theta_{\eta}\left(\frac{(t+s) / 2-t_{0}}{\Delta t}\right) \theta_{h}(t-s) \prod_{i=1}^{N} \theta_{h}\left(x_{i}-y_{i}\right) d Q \\
\leq & \int_{Q}\left|u(s, y)-u\left(t_{0}, y\right)\right| \theta_{\eta}\left(\frac{(t+s) / 2-t_{0}}{\Delta t}\right) \theta_{h}(t-s) \prod_{i=1}^{N} \theta_{h}\left(x_{i}-y_{i}\right) d Q \\
& +\int_{Q}\left|u\left(t_{0}, y\right)-u\left(t_{0}, x\right)\right| \theta_{\eta}\left(\frac{(t+s) / 2-t_{0}}{\Delta t}\right) \theta_{h}(t-s) \prod_{i=1}^{N} \theta_{h}\left(x_{i}-y_{i}\right) d x d y d t d s \\
& +\int_{Q}\left|u_{\varepsilon}\left(t_{0}, x\right)-u_{\varepsilon}(t, x)\right| \theta_{\eta}\left(\frac{(t+s) / 2-t_{0}}{\Delta t}\right) \theta_{h}(t-s) \prod_{i=1}^{N} \theta_{h}\left(x_{i}-y_{i}\right) d Q \\
& -\int_{Q}\left|u(s, y)-u_{\varepsilon}(t, x)\right| \theta_{\eta}\left(\frac{(t+s) / 2-t_{0}}{\Delta t}\right) \theta_{h}(t-s) \prod_{i=1}^{N} \theta_{h}\left(x_{i}-y_{i}\right) d Q \\
\equiv & J+K+L^{\varepsilon}+I_{0} .
\end{aligned}
$$

At the left-hand side, we have

$$
\begin{aligned}
-\int_{Q} \mid & u\left(t_{0}, x\right)-u_{\varepsilon}\left(t_{0}, x\right) \mid \theta_{\eta}\left(\frac{(t+s) / 2-t_{0}}{\Delta t}\right) \theta_{h}(t-s) \prod_{i=1}^{N} \theta_{h}\left(x_{i}-y_{i}\right) d Q \\
= & -\int_{x}\left|u\left(t_{0}, x\right)-u_{\varepsilon}\left(t_{0}, x\right)\right| \int_{y} \prod_{i=1}^{N} \theta_{h}\left(x_{i}-y_{i}\right) \int_{t} \int_{s} \theta_{\eta}\left(\frac{(t+s) / 2-t_{0}}{\Delta t}\right) \theta_{h}(t-s) d Q \\
= & -\frac{1}{2}\left\|u\left(t_{0}, \cdot\right)-u_{\varepsilon}\left(t_{0}, \cdot\right)\right\|_{L^{1}\left(\mathbb{R}_{x}^{N}\right)} .
\end{aligned}
$$

At the right-hand side, we have the following:

$$
\begin{aligned}
J & =1 \cdot \iint_{t_{0}}^{t_{1}}\left\|u(s, \cdot)-u\left(t_{0}, \cdot\right)\right\|_{L^{1}\left(\mathbb{R}_{y}^{N}\right)} \theta_{\eta}\left(\frac{(t+s) / 2-t_{0}}{\Delta t}\right) \theta_{h}(t-s) d s d t \\
& \leq \iint_{t_{0}}^{t_{1}}\left|t_{0}-s\right|\|u(\xi, \cdot)\|_{\mathrm{BV}\left(\mathbb{R}_{+} ; L^{1}\left(\mathbb{R}_{y}^{N}\right)\right)} \theta_{\eta}\left(\frac{(t+s) / 2-t_{0}}{\Delta t}\right) \theta_{h}(t-s) d s d t, \quad s \leq \xi \leq t_{0}, \\
& \leq h\left\|u_{0}(\cdot)\right\|_{\mathrm{BV}\left(\mathbb{R}_{t}^{+} ; L^{1}\left(\mathbb{R}^{N}\right)\right)} \iint_{t_{0}}^{t_{1}} \theta_{\eta}\left(\frac{(t+s) / 2-t_{0}}{\Delta t}\right) \theta_{h}(t-s) d s d t \\
& \leq \frac{h}{2}\left\|u_{0}\right\|_{\mathrm{BV}\left(\mathbb{R}_{t} ; L^{1}\left(\mathbb{R}^{N}\right)\right)},
\end{aligned}
$$


12 Error bounds to conservation laws by kinetic approaches

using Lemma 4.1. We also have

$$
\begin{aligned}
K & \leq \int_{z} \int_{y}\left|u\left(t_{0}, y\right)-u\left(t_{0}, y+z\right)\right| \prod_{i=1}^{N} \theta_{h}\left(z_{i}\right) d z d y \iint_{t_{0}}^{t_{1}} \theta_{\eta}\left(\frac{(t+s) / 2-t_{0}}{\Delta t}\right) \theta_{h}(t-s) d t d s \\
& \leq h \int_{y} \frac{\left|u\left(t_{0}, y\right)-u\left(t_{0}, y+z\right)\right|}{|z|} d y \int_{z} \prod_{i=1}^{N} \theta_{h}\left(z_{i}\right) d z \times \frac{1}{2} \\
& \leq \frac{h}{2}\|u(\cdot)\|_{\mathrm{BV}\left(\mathbb{R}^{N}\right)} \leq \frac{h}{2}\left\|u_{0}\right\|_{\mathrm{BV}\left(\mathbb{R}^{N}\right)}
\end{aligned}
$$

using the change of variables

$$
x_{i}-y_{i}=z_{i} \longleftrightarrow d x_{i}=d z_{i} .
$$

Finally,

$$
\begin{aligned}
L^{\varepsilon} & \leq 1 \cdot h\|u(\xi, \cdot)\|_{\mathrm{BV}\left(\mathbb{R}_{t}^{+} ; L^{1}\left(\mathbb{R}_{y}^{N}\right)\right)} \iint_{t_{0}}^{t_{1}} \theta_{\eta}\left(\frac{(t+s) / 2-t_{0}}{\Delta t}\right) \theta_{h}(t-s) d s d t, \quad t_{0} \leq \xi \leq s, \\
& \leq \frac{h}{2}\left\|u_{\varepsilon}\right\|_{\mathrm{BV}\left(\mathbb{R}_{t} ; L^{1}\left(\mathbb{R}^{N}\right)\right)} \leq \frac{h}{2}\left\|u_{\varepsilon 0}\right\|_{\mathrm{BV}\left(\mathbb{R}_{t} ; L^{1}\left(\mathbb{R}^{N}\right)\right)} .
\end{aligned}
$$

Hence

$$
-\frac{1}{2}\left\|u\left(t_{0}, \cdot\right)-u_{\varepsilon}\left(t_{0}, \cdot\right)\right\|_{L^{1}\left(\mathbb{R}^{N}\right)} \leq h\left\|u_{0}\right\|_{\mathrm{BV}\left(\mathbb{R} ; L^{1}\left(\mathbb{R}^{N}\right)\right)}+\frac{h}{2}\left\|u_{0}\right\|_{\mathrm{BV}\left(\mathbb{R}^{N}\right)}+I_{0} .
$$

For $t=t_{1}$, we consider the following triangle inequality:

$$
\left|u\left(t_{1}, x\right)-u_{\varepsilon}\left(t_{1}, x\right)\right| \leq\left|u\left(t_{1}, x\right)-u\left(t_{1}, y\right)\right|+\left|u\left(t_{1}, y\right)-u(s, y)\right|+\left|u(s, y)-u_{\varepsilon}(t, x)\right| .
$$

With the same techniques, we obtain

$$
\begin{aligned}
\int_{Q} \mid & u\left(t_{1}, x\right)-u_{\varepsilon}\left(t_{1}, x\right) \mid \theta_{\eta}\left(\frac{(t+s) / 2-t_{1}}{\Delta t}\right) \theta_{h}(t-s) \prod_{i=1}^{N} \theta_{h}\left(x_{i}-y_{i}\right) d Q \\
= & \frac{1}{2}\left\|u\left(t_{1}, x\right)-u_{\varepsilon}\left(t_{1}, x\right)\right\|_{L^{1}\left(\mathbb{R}^{N}\right)} \leq h\left\|u_{0}\right\|_{\mathrm{BV}\left(\mathbb{R} ; L^{1}\left(\mathbb{R}^{N}\right)\right)}+\frac{h}{2}\left\|u_{0}\right\|_{\mathrm{BV}\left(\mathbb{R}^{N}\right)}+I_{1} .
\end{aligned}
$$

We sum up by

$$
\begin{gathered}
\left\|u\left(t_{1}, x\right)-u_{\varepsilon}\left(t_{1}, x\right)\right\|_{L^{1}\left(\mathbb{R}^{N}\right)}-\left\|u\left(t_{0}, x\right)-u_{\varepsilon}\left(t_{0}, x\right)\right\|_{L^{1}\left(\mathbb{R}^{N}\right)} \\
\leq 4 h\left\|u_{0}\right\|_{\mathrm{BV}\left(\mathbb{R} ; L^{1}\left(\mathbb{R}^{N}\right)\right)}+2 h\left\|u_{0}\right\|_{\mathrm{BV}\left(\mathbb{R}^{N}\right)}+2\left(I_{0}+I_{1}\right) .
\end{gathered}
$$


Theorem 4.3. Suppose that initially $f_{\varepsilon}(0, x, v) \in L^{1}\left(\mathbb{R}_{v} ; \mathrm{BV}\left(\mathbb{R}^{N}\right)\right)$, which is compactly supported in $v$, and that $f_{\varepsilon}(0, x, v) \equiv f_{0}(x, v)=\chi_{u_{0}(x)}(v)$. Let $u(s, y)$ be the entropy solution of (1.1)-(1.3), and $u_{\varepsilon}(t, x)$ the approximate solution given by (2.3). Then for any $T>0$, and $t_{0}, t_{1}$ in $[0, T]$, the following $L_{x}^{1}$ error bound

$$
\left\|u\left(t_{1}, \cdot\right)-u_{\varepsilon}\left(t_{1}, \cdot\right)\right\|_{L^{1}\left(\mathbb{R}^{N}\right)} \leq\left\|u\left(t_{0}, \cdot\right)-u_{\varepsilon}\left(t_{0}, \cdot\right)\right\|_{L^{1}\left(\mathbb{R}^{N}\right)}+C \sqrt{\varepsilon}
$$

holds true, with $C$ being a positive constant independent on $\varepsilon$.

Proof. From Proposition 3.6, we have

$$
I_{0}+I_{1}=I \leq M \frac{\varepsilon}{h}\left\|u_{0}\right\|_{\mathrm{BV}\left(\mathbb{R}_{x}^{N}\right)},
$$

where $M$ is given by (3.29). Hence

$$
\begin{aligned}
& \left\|u\left(t_{1}, \cdot\right)-u_{\varepsilon}\left(t_{1}, \cdot\right)\right\|_{L^{1}\left(\mathbb{R}^{N}\right)}-\left\|u\left(t_{0}, \cdot\right)-u_{\varepsilon}\left(t_{0}, \cdot\right)\right\|_{L^{1}\left(\mathbb{R}^{N}\right)} \\
& \quad \leq\left(4\left\|u_{0}\right\|_{\mathrm{BV}\left(\mathbb{R} ; L^{1}\left(\mathbb{R}^{N}\right)\right)}+2\left\|u_{0}\right\|_{\mathrm{BV}\left(\mathbb{R}^{N}\right)}\right) h+M \frac{\varepsilon}{h}\left\|u_{0}\right\|_{\mathrm{BV}\left(\mathbb{R}_{x}^{N}\right)} .
\end{aligned}
$$

The optimal choice of $h$ is $h=\sqrt{\varepsilon}$. Then

$$
\left(4\left\|u_{0}\right\|_{\mathrm{BV}\left(\mathbb{R} ; L^{1}\left(\mathbb{R}^{N}\right)\right)}+2\left\|u_{0}\right\|_{\mathrm{BV}\left(\mathbb{R}^{N}\right)}\right) h+M \frac{\varepsilon}{h}\left\|u_{0}\right\|_{\mathrm{BV}\left(\mathbb{R}_{x}^{N}\right)}=C \sqrt{\varepsilon},
$$

where

$$
C=\left\{2+a_{\infty}\left(\sqrt{\varepsilon}+2 N a_{\infty}+2\left(1+a_{\infty}\right)\left|\theta^{\prime}\right|_{L^{1}(\mathbb{R})}\left|\psi_{\eta}\right|_{L^{1}(\mathbb{R})}\right)\right\}\left\|u_{0}\right\|_{\mathrm{BV}\left(\mathbb{R}_{x}^{N}\right)}+4\left\|u_{0}\right\|_{\mathrm{BV}\left(\mathbb{R} ; L^{1}\left(\mathbb{R}^{N}\right)\right)} .
$$

Remark 4.4. Notice that the error is global in time, and optimal in the sense that we cannot have more than $\sqrt{\varepsilon}$, since we use the Kuznetsov techniques.

\section{The parabolic case}

In this short section, we discuss the kinetic approach for the following parabolic case:

$$
\partial_{t} u+\operatorname{div}_{x} A(u)=\Delta u,
$$

where $\Delta=\sum_{i=1}^{N}$ is the Laplacian operator. The corresponding kinetic model is

$$
\left[\partial_{t}+\sum_{i=1}^{N} a_{i}(v) \partial_{x_{i}}\right] f_{\varepsilon}=-\frac{1}{\varepsilon}\left[\chi_{u_{\varepsilon}}-f_{\varepsilon}\right]+\sum_{i=1}^{N} \partial_{x_{i}^{2}} f_{\varepsilon}
$$

which reads as

$$
\left[\partial_{t}+\sum_{i=1}^{N} a_{i}(v) \partial_{x_{i}}-\sum_{i=1}^{N} \partial_{x_{i}^{2}}\right] f_{\varepsilon}=-\frac{1}{\varepsilon}\left[\chi_{u_{\varepsilon}}-f_{\varepsilon}\right] .
$$


14 Error bounds to conservation laws by kinetic approaches

We multiply by $\operatorname{sgn}\left(f_{\varepsilon}-\chi_{\ell}\right)$ and integrate over $v$, we then easily obtain in the weak sense that

$$
\int_{v}\left[\partial_{t}+\sum_{i=1}^{N} a_{i}(v) \partial_{x_{i}}-\sum_{i=1}^{N} \partial_{x_{i}^{2}}\right]\left|f_{\varepsilon}-\chi_{\ell}\right| d v=-\frac{1}{\varepsilon}\left[\int_{v}\left|f_{\varepsilon}-\chi_{\ell}\right|-\left|u_{\varepsilon}-\ell\right|\right] \leq 0
$$

We consider here $\left|f_{\varepsilon}-\chi_{\ell}\right|$ as the "kinetic entropies" for (5.2) in the sense that

$$
-\int_{Q} \int_{v}\left(\left[\partial_{t}+\sum_{i=1}^{N} a_{i}(v) \partial_{x_{i}}-\sum_{i=1}^{N} \partial_{x_{i}^{2}}\right] \varphi\right)\left|f_{\varepsilon}-\chi_{\ell}\right| d v d Q \leq 0 .
$$

We do the same analysis as in Sections 3 and 4. The error found is the same. Notice the following remainder:

$$
\begin{aligned}
\left|\varepsilon \int_{v} \int_{Q_{i=1}} \sum_{i=1}^{N} \partial_{x_{i}}\right| f_{\varepsilon}-\chi_{\ell}\left|\partial_{x_{i}}(\varphi) d v d Q\right| & \leq\left(\varepsilon \sum_{i=1}^{N} \|\left.\partial_{x_{i_{0}}} \cdot f_{\varepsilon}\right|_{L^{\infty}}\right)\left(\sum_{i=1}^{N}\left|\varphi_{x_{i}}\right|_{L^{1}(t, x)}\right) \\
& \leq\left(\varepsilon N\left\|\partial_{x_{i_{0}}} f_{\varepsilon}\right\|_{L^{\infty}}\right)\left(\|\varphi\|_{L^{1}\left(\mathbb{R} ; W^{1}, 1\left(\mathbb{R}^{N}\right)\right)}\right),
\end{aligned}
$$

where $x_{i_{0}}, i_{0} \in 1, \ldots, N$, is the space variable so that $\left|\partial x_{i} f\right| \leq\left|\partial x_{i_{0}} f\right|$.

Remark 5.1. It would be interesting to study the kinetic approach to the case of parabolichyperbolic problems in the sense of Chen and Karlsen in [7] and Chen and Perthame in $[8]$.

\section{Acknowledgment}

The author is thankful to Professor J. P. Vila who introduced him to kinetic and conservation law problems when guiding him during his thesis.

\section{References}

[1] P. L. Bhatnagar, E. P. Gross, and M. Krook, A model for collision processes in gases, Physical Review 94 (1954), no. 3, 511-525.

[2] F. Bouchut, F. Golse, and M. Pulvirenti, Kinetic Equations and Asymptotic Theory, Series in Applied Mathematics (Paris), vol. 4, Gauthier-Villars, Paris, 2000.

[3] F. Bouchut and B. Perthame, Kružkov's estimates for scalar conservation laws revisited, Transactions of the American Mathematical Society 350 (1998), no. 7, 2847-2870.

[4] Y. Brenier, Résolution d'équations d'évolution quasilinéaires en dimension $N$ d'espace à l'aide d'équations linéaires en dimension N + 1, Journal of Differential Equations 50 (1983), no. 3, 375390.

[5] _ Averaged multivalued solutions for scalar conservation laws, SIAM Journal on Numerical Analysis 21 (1984), no. 6, 1013-1037.

[6] C. Cercignani, Mathematical Methods in Kinetic Theory, 2nd ed., Plenum Press, New York, 1984.

[7] G.-Q. Chen and K. H. Karlsen, $L^{1}$-framework for continuous dependence and error estimates for quasilinear anisotropic degenerate parabolic equations, Transactions of the American Mathematical Society 358 (2006), no. 3, 937-963. 
[8] G.-Q. Chen and B. Perthame, Well-posedness for non-isotropic degenerate parabolic-hyperbolic equations, Annales de l'Institut Henri Poincaré. Analyse Non Linéaire 20 (2003), no. 4, 645668.

[9] Y. Giga and T. Miyakawa, A kinetic construction of global solutions of first order quasilinear equations, Duke Mathematical Journal 50 (1983), no. 2, 505-515.

[10] S. N. Kružkov, First order quasilinear equations in several independent variables, Mathematics of the USSR, Sbornik 10 (1970), 217-243.

[11] N. N. Kuznetsov, Accuracy of some approximate methods for computing the weak solutions of a first-order quasi-linear equation, USSR Computational Mathematics and Mathematical Physics 16 (1976), no. 6, 105-119.

[12] N. N. Kuznetsov and S. A. Voloshin, On monotone difference approximations for a first-order quasi-linear equation, Soviet Mathematics. Doklady 17 (1976), 1203-1206.

[13] P.-L. Lions, B. Perthame, and E. Tadmor, A kinetic formulation of multidimensional scalar conservation laws and related equations, Journal of the American Mathematical Society 7 (1994), no. 1, 169-191.

[14] T. Miyakawa, A kinetic approximation of entropy solutions of first order quasilinear equations, Recent Topics in Nonlinear PDE (Hiroshima, 1983), North-Holland Math. Stud., vol. 98, NorthHolland, Amsterdam, 1984, pp. 93-105.

[15] A. Omrane, Estimations d'erreur pour les lois de conservation scalaires par approches cinétiques, et étude du cas des conditions aux limites, Ph.D. thesis, Université de Nice-Sophia Antipolis, 1998.

[16] B. Perthame and E. Tadmor, A kinetic equation with kinetic entropy functions for scalar conservation laws, Communications in Mathematical Physics 136 (1991), no. 3, 501-517.

[17] J. Smoller, Shock Waves and Reaction-Diffusion Equations, Fundamental Principles of Mathematical Science, vol. 258, Springer, New York, 1983.

[18] J.-P. Vila, Convergence and error estimates in finite volume schemes for general multidimensional scalar conservation laws. I. Explicit monotone schemes, Mathematical Modelling and Numerical Analysis 28 (1994), no. 3, 267-295.

A. Omrane: Département de Mathématiques et Informatique, Université des Antilles et de la Guyane, Campus de Fouillole, 97159 Pointe à Pitre Cedex, Guadeloupe, France

E-mail address: aomrane@univ-ag.fr 


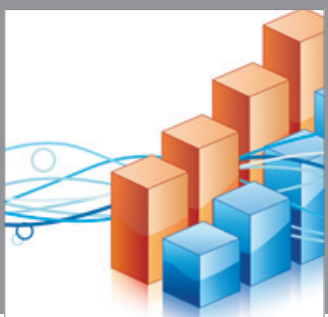

Advances in

Operations Research

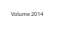

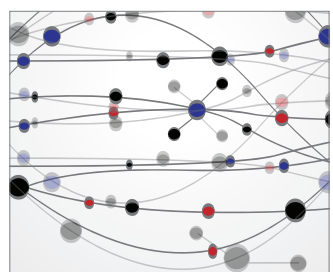

\section{The Scientific} World Journal
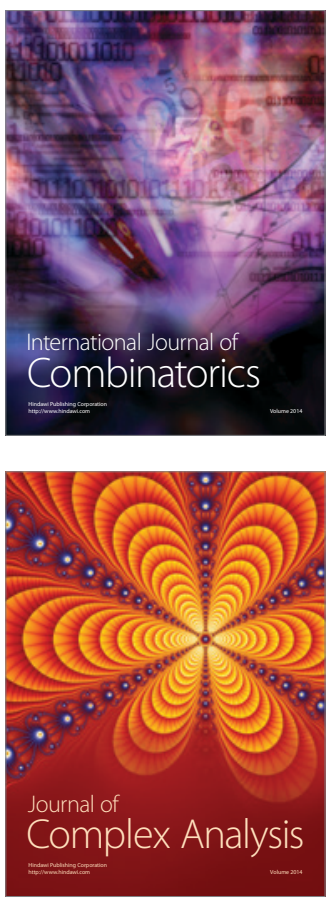

International Journal of

Mathematics and

Mathematical

Sciences
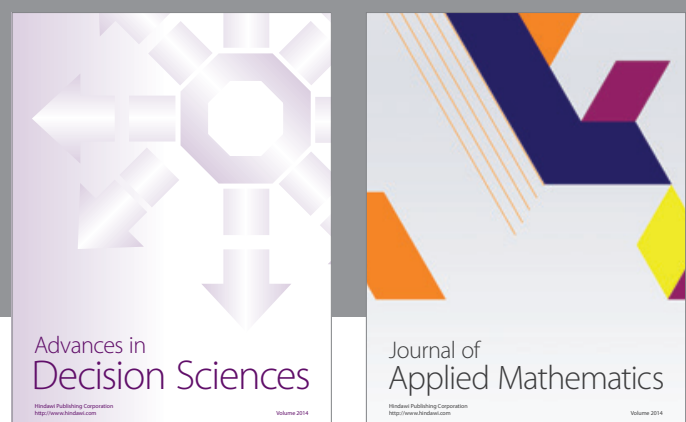

Journal of

Applied Mathematics
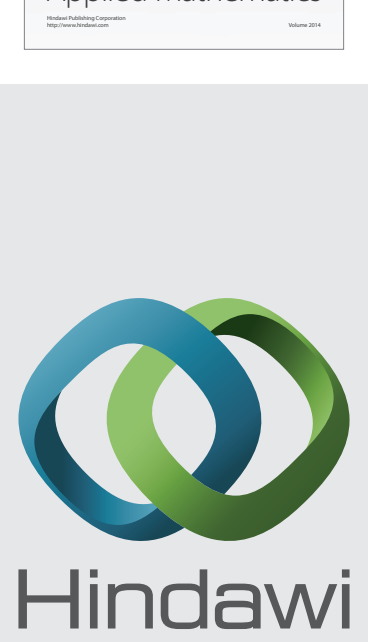

Submit your manuscripts at http://www.hindawi.com
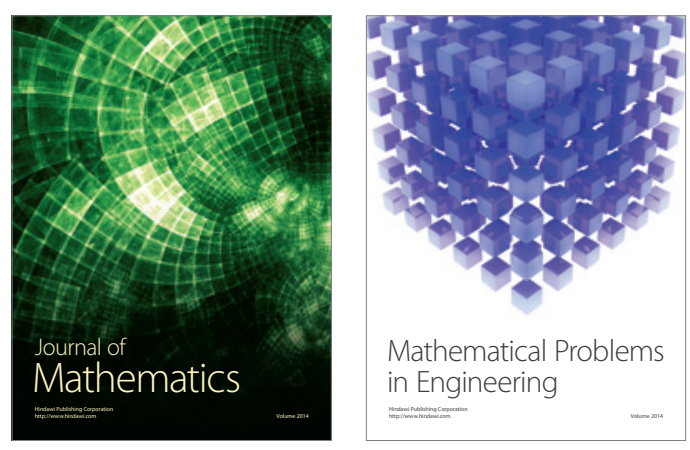

Mathematical Problems in Engineering
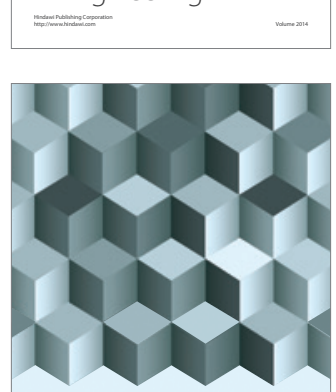

Journal of

Function Spaces
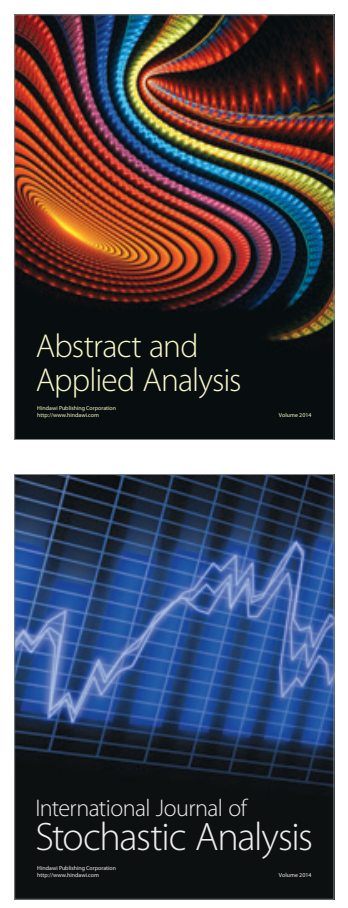

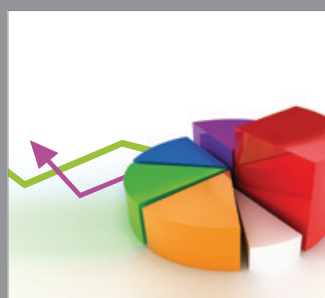

ournal of

Probability and Statistics

Promensencen
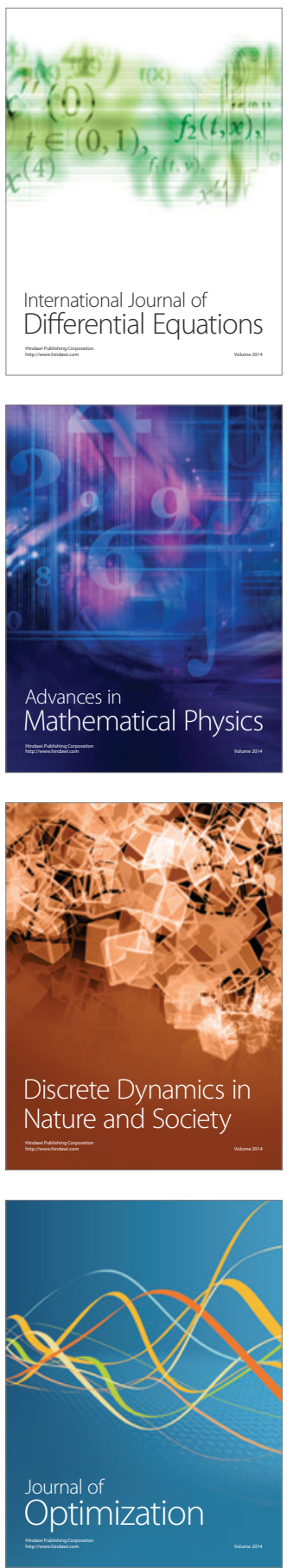